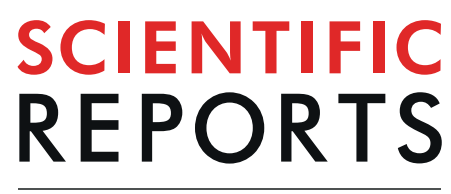

natureresearch

Check for updates

\title{
Larger, but not better, motor adaptation ability inherent in medicated Parkinson's disease patients revealed by a smart- device-based study
}

\author{
Ken Takiyama ${ }^{1 凶}$, Takeshi Sakurada $\mathbb{B}^{2,3}{ }^{2}$ Masahiro Shinya ${ }^{4}$, Takaaki Sato $^{5}$, Hirofumi Ogihara ${ }^{6}$ \\ \& Taiki Komatsu $\mathbb{B}^{7}$
}

Generating appropriate motor commands is an essential brain function. To achieve proper motor control in diverse situations, predicting future states of the environment and body and modifying the prediction are indispensable. The internal model is a promising hypothesis about brain function for generating and modifying the prediction. Although several findings support the involvement of the cerebellum in the internal model, recent results support the influence of other related brain regions on the internal model. A representative example is the motor adaptation ability in Parkinson's disease (PD) patients. Although this ability provides some hints about how dopamine deficits and other PD symptoms affect the internal model, previous findings are inconsistent; some reported a deficit in the motor adaptation ability in PD patients, but others reported that the motor adaptation ability of PD patients is comparable to that of healthy controls. A possible factor causing this inconsistency is the difference in task settings, resulting in different cognitive strategies in each study. Here, we demonstrate a larger, but not better, motor adaptation ability in PD patients than in healthy controls while reducing the involvement of cognitive strategies and concentrating on implicit motor adaptation abilities. This study utilizes a smart-device-based experiment that enables motor adaptation experiments anytime and anywhere with less cognitive strategy involvement. The PD patients showed a significant response to insensible environmental changes, but the response was not necessarily suitable for adapting to the changes. Our findings support compensatory cerebellar functions in PD patients from the perspective of motor adaptation.

Motor adaptation is an essential brain function that modifies motor commands to achieve desired movements in novel situations, such as adapting to use new tools or correcting a movement error. A promising hypothesis about motor adaptation is the internal model hypothesis, which considers the cerebellum to play a role not only in predicting future states of the environment and the body but also in modifying the prediction ${ }^{1}$. Appropriate motor commands can be generated through the outcome predicted by the internal model. To investigate the ability to update the internal model, a motor adaptation paradigm is used ${ }^{2,3}$. In this paradigm, the environment changes through artificially applied perturbations. The subjects thus need to update their internal models to achieve the desired movements while compensating for the perturbation.

${ }^{1}$ Tokyo University of Agriculture and Technology, Department of Electrical Engineering and Computer Science, 2-24-16, Nakacho, Koganei, Tokyo, Japan. ${ }^{2}$ Ritsumeikan University, College of Science and Engineering, 1-1-1, Nojihigashi, Kusatsu, Shiga, Japan. ${ }^{3}$ Jichi Medical University, Department of Neurosurgery, 3311-1, Yakushiji, Shimotsuke, Tochigi, Japan. ${ }^{4}$ Hiroshima University, Graduate School of Humanities and Social Sciences, Kagamiyama 1-7-1, Higashi-Hiroshima, Hiroshima, Japan. ${ }^{5}$ JA Nagano Koseiren Kakeyu-Misayama Rehabilitation Center Kakeyu Hospital, Department of Physical Therapy, 1308, Kakeyuonsen, Ueda, Nagano, Japan. ${ }^{6}$ Japan University of Health Sciences, Department of Physical Therapy, 2-555, Hirasuka, Satte, Saitama, Japan. ${ }^{7}$ Nihon University, College of Sports Science, 3-34-1 Shimouma, Setagaya, Tokyo, Japan. ${ }^{\circledR e}$-mail: t.j.ken.takiyama@gmail.com 
The cerebellum can play crucial roles in the internal model ${ }^{1}$, which has been supported via deficit motor adaptation abilities in cerebellar ataxia patients ${ }^{4,5}$. In addition to cerebellar ataxia patients, Parkinson's disease (PD) patients also showed impaired sensorimotor adaptation abilities, such as adapting to 90 degree visuomotor rotation $^{6}$ or adapting to three-dimensional arm-reaching movements ${ }^{7}$. PD, the second most common degenerative neurological disease, causes a lack of dopamine neurons in the substantia nigra pars compacta. Due to the impaired motor adaptation ability in PD patients, the internal model can be affected by not only the cerebellum but also other brain regions. The motor adaptation ability in PD patients can provide some hints to deepen our knowledge about the internal model.

To investigate the motor adaptation ability inherent in PD patients, previous studies relied on a constant amount of perturbation that was applied abruptly at a specific time (we refer to this type of perturbation as abrupt perturbation hereafter). To adapt to the abrupt perturbation, subjects tend to rely on explicit strategies or their cognitive abilities $^{8,9}$. For example, when the perturbation was 90 degree visuomotor rotation that caused a 90 degree deviation in the movement angle between the actual and perturbed movements, it was possible to achieve the desired movements by aiming at the 90 degree location distant from a target. With an abrupt perturbation, subjects tend to notice the onset of the perturbation or task switching ${ }^{9-11}$. In the motor adaptation to the abrupt perturbation, it is difficult to determine whether the impaired sensorimotor adaptation in PD patients is caused by their cognitive abilities (i.e., the influence of the task switch) or adaptation abilities.

In contrast to the adaptation to an abrupt perturbation, PD patients showed a compatible adaptation ability with healthy elderly individuals in responding to a gradually applied visuomotor transformation (we refer to this type of perturbation as a gradual perturbation hereafter $)^{12,13}$. In contrast to abrupt perturbations, a striking feature of gradual perturbations is their difficulty in being noticed ${ }^{9-11,14,15}$. A task switch can thus be related to an adaptation to the abrupt perturbation and not to an adaptation to the gradual perturbation. The influence of the task switch can be a candidate for interpreting the compatible adaptation ability of PD patients with healthy elderly individuals in adapting to only the gradual perturbation.

Additional support for the influence of task switching on motor adaptation in PD patients is the lack of savings in PD patients. Young individuals and healthy elderly individuals show faster adaptation in re-adaptation trials than in the initial adaptation trials, such as in the A-B-A paradigm, which is referred to as savings ${ }^{16-18}$. In contrast, PD patients do not exhibit savings ${ }^{19,20}$. Because task switching can lead to savings, the adaptation ability inherent in $\mathrm{PD}$ patients can be influenced by task switching.

Because task switching is a candidate in affecting the motor adaptation ability in PD patients, it is necessary to exclude the influence of task switching in detail. Although a gradual perturbation can involve less task switching than an abrupt perturbation, previous studies used 60 degree visuomotor rotation ${ }^{12}$ or a $7.8 \mathrm{~cm}$ transformation in $10 \mathrm{~cm}$ arm-reaching movements in total ${ }^{13}$. These perturbations can involve the influence of task switching because the aiming direction can deviate from the target location even if the gradual perturbation involves more than a 45 degree visuomotor rotation (Fig. $3 \mathrm{in}^{9}$ ). It is thus still unclear whether the adaptation ability is still compatible between PD patients and elderly individuals because the task switch can be involved in a gradual perturbation with a large amplitude.

Here, we investigated the motor adaptation ability of PD patients while decreasing the influence of the task switch as much as possible. To reduce the influence of the task switch, we relied on a gradually applied perturbation whose existence was noticeable by 1 out of the 82 participants in our previous study ${ }^{11}$; the visuomotor rotation changed by one degree in each trial, and the maximum value of the rotation was 15 degrees. Based on a previous study, task switches are less involved in gradually applied 15-degree visuomotor rotation than in gradually applied 45-degree visuomotor rotation ${ }^{9}$. We demonstrate that the PD patients show a larger, but not better, motor adaptation ability than elderly individuals and young individuals, rather than a compatible or impaired ability.

In addition to reducing the influence of the task switch, we also decreased the burden of participating in the motor adaptation experiments. Almost all the previous experiments on motor adaptation relied on manipulanda or pen tablet settings. These settings require the subjects to travel to the laboratory, which can be a burden, especially for patients, to participate in the experiments. To minimize this burden, we utilize a smart-device-based experimental setting that is available to conduct motor adaptation experiments anytime and anywhere ${ }^{11}$. Smart-device-based experiments have been proposed to conveniently investigate motor adaptation or visuomotor abilities for flexible applications $s^{21,22}$. Our smart-device-based setting has been validated under several conditions and by comparing it to a conventional experimental setting with manipulanda ${ }^{11}$. We refer to our experimental setting as the POrtable Motor learning LABoratory (PoMLab). The PoMLab can decrease the burden for the participants, PD patients, elderly individuals, and young individuals by removing the need to go to a specific place at a particular time. In this study, we demonstrate a larger, but not better, motor adaptation inherent in PD patients using our PoMLab setting.

\section{Methods}

Participants. Fifty-four subjects participated in the current study; their ages and sexes are summarized in Table 1 . This study was approved by the ethics committees of the Tokyo University of Agriculture and Technology, Jichi Medical University, and Kakeyu Hospital. The PoMLab experiments were conducted while each participant was seated on a chair and an experimenter was present and close to the participant. The current study was performed in accordance with the relevant guidelines and regulations in the Declaration of Helsinki. All participants provided written informed consent before the start of the experiments.

The PD patients were outpatients satisfying the following inclusion criteria. The elderly individuals were inpatients with broken lower-limb bones that did not disturb performance in our study. The young individuals were volunteers. All of the participants were naive to the purpose of the study. 


\begin{tabular}{|l|l|l|l|l|}
\hline & PD patients & Elderly individuals & p-value & Young individuals \\
\hline Age & $70.06 \pm 7.34$ & $75.67 \pm 12.70$ & 0.1138 & $21.33 \pm 1.50$ \\
\hline UPDRS & $26.06 \pm 12.07$ & N/A & N/A & N/A \\
\hline H \& Y & $2.67 \pm 0.69$ & N/A & N/A & N/A \\
\hline MMSE & $27.78 \pm 2.07$ & $27.72 \pm 2.45$ & 0.9418 & N/A \\
\hline Duration (year) & $6.72 \pm 4.32$ & N/A & N/A & N/A \\
\hline Sex & $\mathrm{M}=9, \mathrm{~F}=9$ & $\mathrm{M}=8, \mathrm{~F}=10$ & $\mathrm{~N} / \mathrm{A}$ & $\mathrm{M}=16, \mathrm{~F}=2$ \\
\hline
\end{tabular}

Table 1. Attributes of the participants. The "p-values" in this table indicate the p-values from a two-sample t-tests between the PD patients and the elderly individuals. "M" and "F" under sex indicate male and female, respectively. All the values in this table denote the means \pm standard deviations.

PD was diagnosed clinically according to the UK PD Society Brain Bank criteria. All of the patients had bradykinesia and at least one of the three features of PD: 4-6 Hz resting tremor, rigidity, and postural instability. All of the PD patients had asymmetric onset and showed a positive response to dopaminergic medication. None exhibited atypical symptoms such as severe gaze palsy or symptomatic dysautonomia. All PD patients participated in the experiment approximately 1-3 hours after the last levodopa administration and were in an ON state without troublesome dyskinesia while performing the experimental task.

The PD patients were clinically evaluated based on the Unified Parkinson's Disease Rating Scale (UPDRS) ${ }^{23}$ and the adapted version of the Hoehn and Yahr scale (H\&Y) ${ }^{24}$. The Mini-Mental State Examination (MMSE) score was used to evaluate cognitive abilities. Table 1 summarizes the ages, UPDRS scores, H\&Y scores, MMSE scores, duration, and sex in each group, if available.

Inclusion criteria. PD patients and elderly individuals whose MMSE scores were greater than 22 [no significant difference, two-sample t-test $\mathrm{p}=0.9418$ ], indicating no severe cognitive decline, were included in the study. The minimum value of MMSE in the PD patients was $24(\mathrm{~N}=1)$, and that in the elderly was $23(\mathrm{~N}=1)$. The second and third minimum values of MMSE in the PD patients were $25(\mathrm{~N}=2)$ and $26(\mathrm{~N}=3)$, respectively, and those in the elderly were $24(\mathrm{~N}=2)$ and $25(\mathrm{~N}=1)$. Our results were invariant when we set the MMSE criteria to be 23 or 24 because the MMSE in the majority of the participants was greater than 25 . Furthermore, the included PD patients were on medication, resulting in no tremor. There were no musculoskeletal and visual impairments that inhibited performing the required tasks in the current study.

Smart device. We used an Android tablet (Nexus 9, HTC, Taipei City, Taiwan, 20481536 pixels, 228.25153.687.95 $\mathrm{mm}$ screen size, and $436 \mathrm{~g}$ weight) throughout our experiments.

PoMLab application. The PoMLab application was developed using a personal edition of Unity (version 5.2). The PoMLab application is available on our GitHub page (https://github.com/masahiroshinya/PoMLab).

PoMLab settings. The cursor position on the tablet display $\left(d_{x}, d_{y}\right)$ that was controlled by participants was determined as follows. First, the cursor position in the tablet coordinate system, $\left(p_{x}, p_{y}\right)$, was determined based on the measured acceleration of the tilting motions in the $\mathrm{x}$ - and $\mathrm{y}$-axes of the tablet coordinate system $\left(a_{x}, a_{y}\right)$ through a low-pass filter; $p_{x}=0.95 p_{x}+0.05 \arcsin \left(a_{x}\right)-o_{x}$ and $p_{y}=0.95 p_{y}+0.05 \arcsin \left(a_{y}\right)-o_{y}$, where $o_{x}$ and $o_{y}$ are offsets to determine the initial cursor position in each trial $\left(o_{x}=0\right.$ and $\left.o_{y}=-30\right)$. The $a_{x}$ and $a_{y}$ were sampled at $200 \mathrm{~Hz}$. The cursor position in the accelerometer coordinate system was transformed into the position on the tablet display $\left(d_{x}, d_{y}\right)$ by multiplying by the rotation matrix $R ;\left(d_{x}, d_{y}\right)^{T}=R\left(p_{x}, p_{y}\right)^{T}$, where ()$^{T}$ denotes the transpose of the vector. Without any visuomotor rotation, the cursor position in the accelerometer coordinate system corresponded to that in the display coordinate system. In the $t$ th adaptation trial, visuomotor rotation was applied through the rotation angle $p_{t}$. The detailed settings and validations of PoMLab are provided in our previous study ${ }^{11}$, and our code is available on GitHub (https://github.com/masahiroshinya/PoMLab).

Experimental procedures. The required task was to tilt the held tablet device appropriately. Corresponding to the tilting motion, the cursor displayed on the tablet moved (a yellow circle with a $4.5 \mathrm{~mm}$ radius on Nexus 9). The participants were instructed to move the cursor toward the visually instructed target (a purple circle with a $4.5 \mathrm{~mm}$ radius on Nexus 9) on the display in a straightforward manner within two seconds (Fig. 1A). At the beginning of each trial, the subjects needed to tilt the tablet to set the cursor at the initial position in the center of the tablet screen (a blue circle with a $9.0 \mathrm{~mm}$ radius on Nexus 9) for 1 second. After 1 second, the color of the initial position changed from blue to red, and the target appeared. Because the target was displayed for two seconds, the subjects needed to tilt the tablet to hit the target within these two seconds. The cursor, target, and initial position were displayed on the tablet screen, and the cursor moved according to the tilting motion, which enabled the motor adaptation experiments to be conducted solely with the tablet device.

The subjects participated in 20 practice trials and 80 adaptation trials. In the first 20 practice trials, the target position was pseudorandomly set to either $60,75,90,105$, or 120 degrees without any visuomotor rotation (90 degrees indicated the 12 o'clock position on the tablet display). In the following 80 adaptation trials, the target position was fixed at 90 degrees. The adaptation trials were divided into two parts. In the first 40 adaptation trials, the subjects experienced gradually increasing and vanishing clockwise (CW) perturbations. In the latter 40 trials, the subjects underwent counterclockwise (CCW) perturbation. Half of the participants experienced the CW 

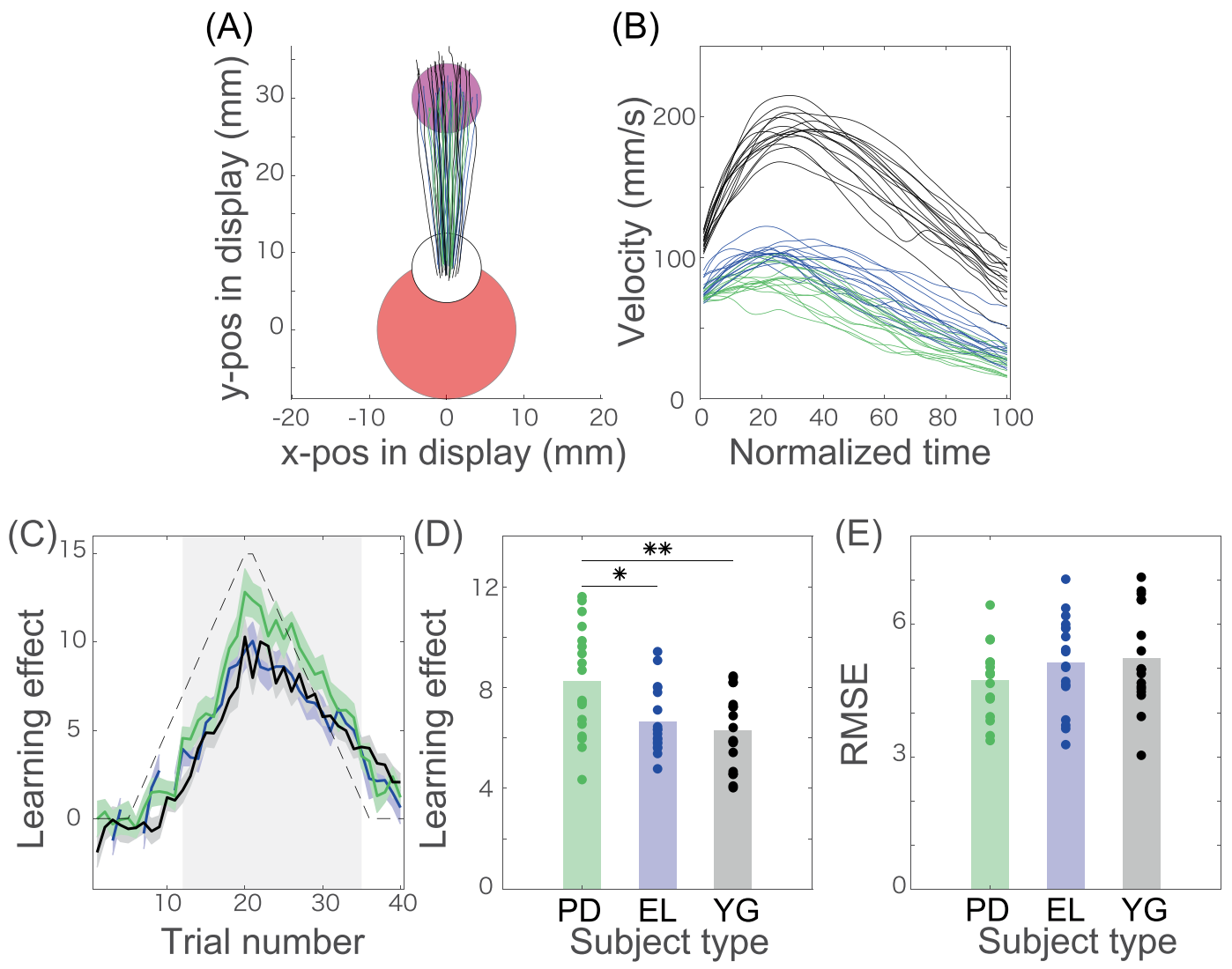

Figure 1. Kinematics and adaptation curve in the PoMLab experiments. (A) Trajectories displayed on the tablet monitor. The green, blue, and solid black lines denote the averaged trajectories across the PD patients, the age- and MMSE-matched elderly individuals, and young individuals, respectively, in every five trials $(\mathrm{N}=18$ in each group). The red, white, and magenta circles indicate the initial position, the controlled cursor, and the target, respectively. (B) Measured velocity. The green, blue, and solid black lines denote the averaged velocities along the $y$-axis across the PD patients, the age- and MMSE-matched elderly individuals, and the young individuals, respectively, in every five trials. (C) Adaptation curves and the perturbation schedule. The horizontal axis denotes the trial number, and the vertical axis indicates the adaptation effects or the degree of perturbation (black dotted line). The adaptation effects were calculated based on the movement angles at the time when the velocity along the $y$-axis reached its maximal value. The green, blue, and solid black lines indicate the adaptation effects averaged across the PD patients, the age- and MMSE-matched elderly individuals, and the young individuals, respectively. The green, blue, and black shaded areas indicate the standard error of the mean for the adaptation effects in each group. The gray shaded area denotes the trial number where adaptation effects in all the groups are significantly different from zero ( $t$-test $\mathrm{p}<0.01$ [corrected]). (D) Adaptation effects averaged across the trials denoted in the gray shaded area in panel (C). Each dot indicates the adaptation effects for each subject. Each bar shows the mean adaptation effects in each group. * and ** indicate statistically significant differences with $\mathrm{p}<0.05$ and $\mathrm{p}<0.01$, respectively (Tukey's post hoc test following one-way ANOVA). (E) RMSE averaged across the trials denoted in the gray shaded area in panel (C).

perturbation first, and the other half of the participants experienced the CCW perturbation first. No subjects were aware of the existence of the perturbation. The experiment typically took less than 30 minutes.

Evaluation of the adaptation effects. The adaptation effects were evaluated depending on the movement angles of the cursor when the velocity on the y-axis reached its peak value (Fig. 1B). The movement angles were defined as the angles between the straight line toward the target and the tangential to the cursor trajectory. The movement trajectories are displayed in Fig. $1 \mathrm{~A}$ after the movement started. The onsets were detected when the velocity along the $y$-axis on the display exceeded the mean +2.5 times the standard deviation calculated in each trial. To avoid evaluating outliers, movement angles in each trial were excluded when these exceeded $\left(15+p_{t}\right)$ when $p_{t} \geq 0$ or $\left(-15+p_{t}\right)$ otherwise. There was no significant difference in the number of excluded trials between the PD patient group [ $3.38 \pm 2.96$ out of 80 trials] and the elderly group [ $4.50 \pm 4.76$ out of 80 trials] $(p=0.6053)$ or between the PD patient group and the young group [1.61 \pm 2.20 out of 80 trials] $(\mathrm{p}=0.2830)$. There was a significant difference between the elderly and young groups $(\mathrm{p}=0.0413)$. We confirmed the invariance of the following results when the exclusion criteria were $\left(c+p_{t}\right)$ when $p_{t} \geq 0$ or $\left(-c+p_{t}\right)$ otherwise with $c=11.5,12$, $13,14,16,20$, or 25 . When $c$ was less than 11.5 , at least one participant in each group showed outliers in the same 
$\operatorname{trial}(\mathrm{s})$ with $p_{t}<0$ and $p_{t} \geq 0$. This disturbed the analysis for the age- and MMSE-matched participants in the PD and elderly groups. We thus focused on the case when $c>11.5$, especially when $c=15$, throughout this study.

Data analysis. Because outliers were detected in some trials based on the above-mentioned criteria, adaptation effects were averaged across the CW and the CCW conditions in each subject to exclude the effects of the outliers. There were no outliers at the same trials in the CW and the CCW conditions in all the subjects; the averaged adaptation effects could be reasonably discussed across all the subjects. Because the effects in the CW conditions took positive values and those in the CCW conditions took negative values, we averaged those by multiplying -1 to the adaptation effects in the CCW condition. If there were outliers at the $k$ th trial in a subject in the CW condition, for example, the adaptation effects at the $k$ th trial corresponded to that in the CCW condition.

The averaged adaptation effects across the CW and the CCW conditions of the ith subject, $x_{i}=\left(x_{1, i}, \ldots, x_{40, i}\right)$, were decomposed into three parameters: the temporal delay $\Delta_{i}\left(\Delta_{i} \geq 0\right)$, the amplitude $A_{i}\left(A_{i} \geq 0\right)$, and the phase $\phi_{i}$. The temporal delay was calculated by temporally sliding the fragments of $x_{i}, x_{i}\left(\Delta_{i}\right)=\left(x_{9+\Delta_{i}, i}, \ldots, x_{32+\Delta_{i} i}\right)$, to minimize the squared error from the fragments of the perturbation sequence $p_{i}=\left(p_{9}, \ldots, p_{32}\right)$. The squared error between the adaptation effects and the perturbation is hereafter referred to as the task error. Of note, the task error corresponds to the prediction error between the predicted and actual movements because the movements toward the target possibly correspond to both the desired and predicted movements with an unnoticeably gradual perturbation. We chose these fragments because the fragments of $x_{i}$ in the PD group were significantly different from 0 when the trial number was between 12 and 35 (i.e., $x_{i}=\left(x_{12, i}, \ldots, x_{35, i}\right)$ were significantly different from 0 , t-test $\mathrm{p}<0.01$ [corrected]) and the squared error between the averaged fragments of the adaptation curve in the $\mathrm{PD}$ patients and the fragments of the perturbation sequence took its minimal value when the fragments were chosen to be $\left(p_{9}, \ldots, p_{32}\right)$. In this case, $\Delta_{\mathrm{i}}$ equaled to three.

After determining $\Delta_{i}^{*}$ as $\Delta_{i}^{*}=\arg \min _{\Delta_{i}}\left(\frac{1}{24} \sum_{j=9}^{32}\left(x_{j+\Delta_{i}, i}-p_{j}\right)^{2}\right)$, we calculated the amplitude $A_{i}$ as $A_{i}=\frac{\left|x_{i}\left(\Delta_{i}^{*}\right)\right|}{|p|}$ and the phase $\phi_{i}$ as $\phi_{i}=\arccos \frac{x_{i}\left(\Delta_{i}^{*}\right) \cdot p}{\left|x_{i}\left(\Delta_{i}^{*}\right)\right||p|}$, where $\left|x_{i}\left(\Delta_{i}^{*}\right)\right|=\sqrt{x_{9+\Delta_{i}^{*}}^{2}+\ldots+x_{32+\Delta_{i}^{*}}^{2}}$ indicates the norm of $x_{i}\left(\Delta_{i}^{*}\right),|p|=\sqrt{p_{9}^{2}+\ldots p_{32}^{2}}$ indicates the norm of $p$, and $x_{i}\left(\Delta_{i}^{*}\right) \cdot p=x_{9+\Delta_{i}^{*}} p_{9}+\ldots+x_{32+\Delta_{i}} p_{32}$ indicates the inner product of $x_{i}\left(\Delta_{i}^{*}\right)$ and $p . \Delta_{i}$ indicates the temporal delay with which each subject minimizes the task error according to the imposed perturbation. $A_{i}$, the amplitude of adaptation effects, indicates the response strength to the applied perturbation. $\phi_{i}$ indicates the similarity between the adaptation effects and the applied perturbation sequence. To quantify the similarity between the adaptation effect and the perturbation while considering the amplitude, the phase, and the temporal delay together, we calculated the root-mean-squared error, $\operatorname{RMSE}_{i}=\sqrt{\frac{1}{24} \sum_{j=9}^{32}\left(x_{j+\Delta_{i}^{*}, i}-p_{j}\right)^{2}}$. To quantify the trajectories, we calculated the trajectory error as the sum of the lateral deviations within each trial: $\mathrm{TE}_{i}=\frac{1}{T} \sum_{f=1}^{T} d_{x, f}$, where $d_{x, f}$ indicates the x-position in the display coordinate system at the $f$ th time frame and $\mathrm{T}$ indicates the total number of time frames within the trial. We calculated these five variables (i.e., $\Delta_{i}^{*}, A_{i}, \phi_{i}, \mathrm{RMSE}_{i}$, and $\mathrm{TE}_{i}$ ) throughout the current study.

Statistical analysis. We utilized one-way ANOVA with a group factor (patients with PD, elderly individuals, and young individuals), followed by Tukey's post hoc tests to compare each group if there was no specified notification about the statistical test. We used MATLAB 2016b (Mathworks, Nantick MA) for all statistical analyses.

\section{Results}

Eighteen PD patients, eighteen age- and MMSE-matched elderly individuals, and eighteen university students (referred to as young individuals in the following) adapted to visuomotor rotation through the PoMLab setting using a tablet device (the age distributions and clinical scores are summarized in Table 1). Figure 1A,B indicate the cursor trajectories and velocities along the $y$-axis, respectively, in the adaptation trials averaged across all the subjects in each group.

We defined the adaptation effects as the movement angle at the time when the velocity along the y-axis took its peak value in each trial. These angles were typical values for the discussion of motor adaptation because we focused on the adaptation to the visuomotor rotation for which the subjects needed to compensate for the perturbation in the movement angles. The movement angles at the timings of peak velocities are thus referred to as adaptation effects hereafter. The angles of visuomotor rotation $p_{t}$ at the $t$ th trial or visuomotor rotation itself are referred to as perturbations hereafter. Although the adaptation to the perturbation can consist of an explicit component (i.e., cognitive ability) and an implicit component (i.e., adaptive component in motor domain) ${ }^{8,9}$, we instructed the participants to aim at the target straightforwardly, which enabled us to exclude the explicit components $^{8,9}$. In addition, no participant was aware of the existence of the perturbation, suggesting that the following results mainly consisted of implicit components rather than explicit components.

The adaptation effects showed between-trial variation depending on the between-trial varying perturbation (Fig. 1C). The shaded area in Fig. 1C denotes the trial numbers (trials 12-35) when the adaptation effects of PD patients were significantly different from 0 ( $\mathrm{t}$-test $\mathrm{p}<0.01$ [corrected]). We compared the adaptation effects averaged across the trials denoted by the shaded area in each subject (Fig. 1D). There was a significant group effect $(\mathrm{F}(2,51)=6.75, \mathrm{p}=0.0025)$, indicating the difference among the PD patients, elderly individuals, and young individuals. There was a significant difference in the adaptation effects between the PD patients $[8.25 \pm 0.51$, mean \pm s.e.m., standard error of the mean] and the elderly individuals $[6.65 \pm 0.31](p=0.0184)$ and between the PD patients and the young individuals $[6.28 \pm 0.36](\mathrm{p}=0.0032)$. However, there was no significant difference in the adaptation effects between the elderly individuals and the young individuals $(p=0.8050)$. In summary, the 
PD patients had adaptation effects approximately $20 \%$ larger than those of the elderly individuals and those of the young individuals in these measures.

This larger adaptation seemed to be related to previous findings whereby PD patients showed a large amount of visual feedback response to minimize visually perceived error ${ }^{25}$ and that the feedback response can be a source of motor adaptation ${ }^{26}$. The larger motor adaptation in PD patients would thus originate from the large visual feedback response. Based on this assumption, we could expect that the PD patients possess better ability to minimize the task error or the prediction error between the aimed target and visual cursor because a common function of both the visual feedback response and motor adaptation is to minimize the error. To investigate the ability to minimize the prediction error, we calculated the root mean squared error (RMSE), a measure of the prediction error between the adaptation effects and the perturbation (a detailed definition of this metric is given in the Methods section). There was no group effect $(\mathrm{F}(2,51)=1.35, \mathrm{p}=0.27)$ and no significant difference among the three groups $(\mathrm{p}>0.28)$ in the RMSE (Fig. 1E). The PD patients thus did not possess better motor adaptation ability to minimize the prediction error compared to the elderly and young individuals, which contrasted with the assumption that the larger motor adaptation would originate from the large visual feedback response. Summarizing, the PD patients showed more substantial adaptation effects than the elderly individuals and young individuals but a comparable ability to minimize the RMSE. In other words, PD patients possess larger, but not better, motor adaptation ability than healthy and elderly individuals.

The adaptation ability inherent in the PD patients was investigated via the average across a certain number of trials rather than the whole adaptation curve. To further examine the adaptation effects in detail, we decomposed the adaptation effects into three components: the amplitude $A$, used to quantify the magnitude of the response to the perturbation; the phase $\phi$, used to quantify the similarity between the adaptation curves and the perturbation; and the temporal delay $\Delta$, used to quantify the temporal sensitivity of the response to the perturbation.

For the amplitude measurement (Fig. 2A), there was a significant group effect $(\mathrm{F}(2,51)=6.76, \mathrm{p}=0.0025)$, a significant difference between the PD patients $[0.910 \pm 0.050]$ and the elderly individuals $[0.752 \pm 0.025]$ $(\mathrm{p}=0.0162)$, a significant difference between the PD patients and the young individuals $[0.721 \pm 0.037]$ $(\mathrm{p}=0.0034)$, and no significant difference between the elderly individuals and the young individuals $(\mathrm{p}=0.842)$. Figure $2 \mathrm{D}$ shows the adaptation curves of the representative $\mathrm{PD}$ patients who showed large (solid magenta lines) and small (solid cyan lines) amplitude values. The PD patients showed an approximately $20 \%$ larger response in amplitude than the elderly individuals and the young individuals.

For the phase measurement (Fig. $2 B)$, there was no significant group effect $(F(2,51)=1.16, p=0.32)$ and no significant difference between each group $(p>0.3104$ among the PD patients $[0.930 \pm 0.009$ ], the elderly individuals $[0.906 \pm 0.014]$, and the young individuals [0.918 \pm 0.008$]$. Figure $2 E$ shows the adaptation curves of the representative PD patients who showed large (solid magenta lines) and small (solid cyan lines) phase values. In contrast to the amplitude, the PD patients showed a comparable phase with the elderly individuals and the young individuals.

For the delay (Fig. 2C), there was a significant group effect $(F(2,51)=3.62, p=0.034)$, no significant difference between the PD patients [2.78 \pm 0.42$]$ and the elderly individuals $[4.11 \pm 0.65],(p=0.18)$, a significant difference between the PD patients and the young individuals [4.72 \pm 0.48$](\mathrm{p}=0.030)$, and no significant difference between the elderly individuals and the young individuals $(\mathrm{p}=0.688)$. Figure $2 \mathrm{~F}$ shows the adaptation curves of the representative PD patients who showed large (solid magenta lines) and small (solid cyan lines) delay values. The PD patients showed smaller response delays compared to the young individuals.

Taken together, the adaptation effects of the PD patients were larger than those of the elderly individuals and the young individuals (Fig. 1C,D, respectively) because the PD patients showed larger amplitudes compared to the other two groups (Fig. 2A). In addition, a slightly faster response delay in the PD patients contributed to the large adaptation effects (Fig. 2C).

We further considered other factors that may affect the larger substantial adaptation effects in PD patients. A candidate influencing the adaptation effects was the movement time. Following previous studies that reported slower movement times in PD patients than in elderly individuals ${ }^{27}$, those patients who participated in our study also showed slower movement times in our experimental setting than the young individuals (Fig. 3A, significant group effect, $\mathrm{F}(2,51)=21.96, \mathrm{p}=1.32 \times 10^{-7}$, no significant difference between the PD patients $[108.77 \pm 6.84 \mathrm{~ms}$ ] and the elderly individuals [95.77 $\pm 8.13 \mathrm{~ms}], \mathrm{p}=0.3321$, a significant difference between the $\mathrm{PD}$ patients and the young individuals $[51.41 \pm 3.27 \mathrm{~ms}], \mathrm{p}=1.93 \times 10^{-7}$, and a significant difference between the elderly individuals and the young individuals, $\mathrm{p}=3.11 \times 10^{-5}$ ). To investigate the possible effects of the movement time on the amplitude, we normalized the movement time within each group so that the mean and the standard deviation of the movement time in each group equaled 0 and 1 , respectively. After normalization, we calculated the correlation coefficients between the amplitude and the grouped and normalized movement times. If the movement time affected the adaptation effects, we could expect some correlation between these metrics. In contrast to this assumption, there was no significant correlation between the magnitude and the normalized movement times (Fig. $3 \mathrm{~B}, \mathrm{r}=0.15, \mathrm{p}=0.27$ ). Additionally, there was no correlation between the phase and the normalized movement time $(\mathrm{r}=0.10, \mathrm{p}=0.46)$ or between the lag and the normalized movement time $(\mathrm{r}=-0.15, \mathrm{p}=0.28)$. These results indicate that the movement time was not a significant factor affecting the adaptation effects.

Although the current study mainly focused on the movement angle at the timing of peak velocity as a measure of motor adaptation, the movement angle could include a certain amount of visual feedback response. The consideration of the feedback response thus enabled a deeper understanding of the larger, but not better, motor adaptation ability inherent to PD patients. Figure 3C shows the relation, indicated by shaded areas, between the TE during the trials, which is a possible factor reflecting a sufficient amount of visual feedback response, and the adaptation effects. If the feedback response was a more dominant factor affecting the motor adaptation ability, we would predict a smaller TE (i.e., larger feedback response) in PD patients than in healthy elderly and young 
(A)
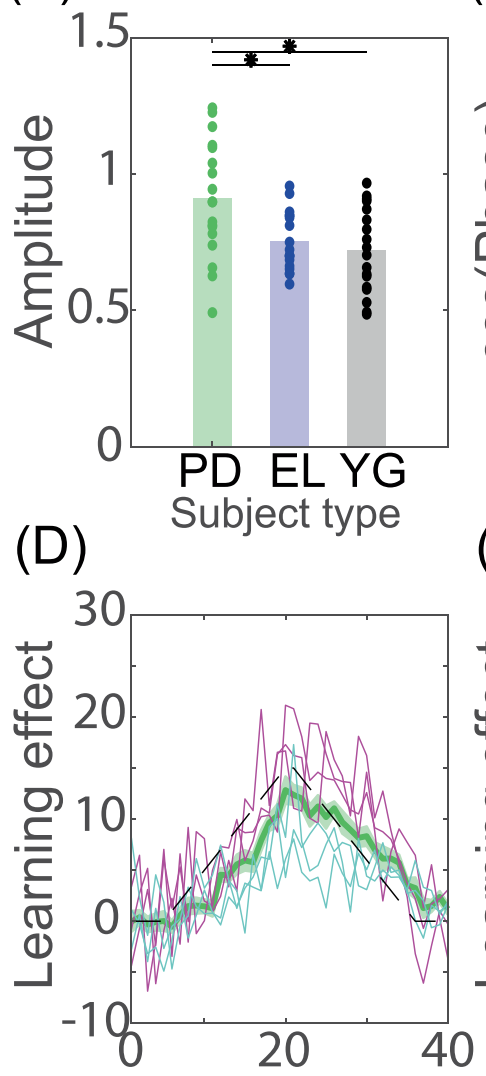

(B)

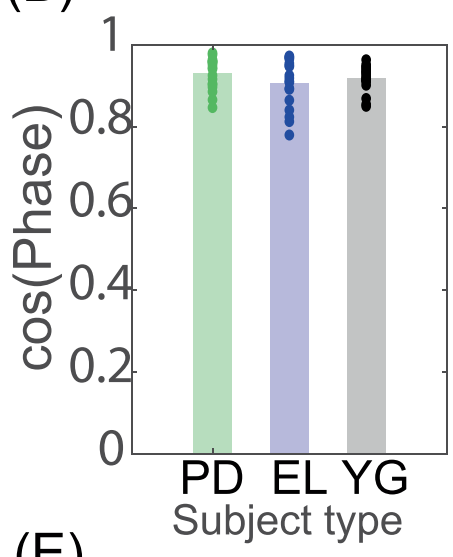

(E)

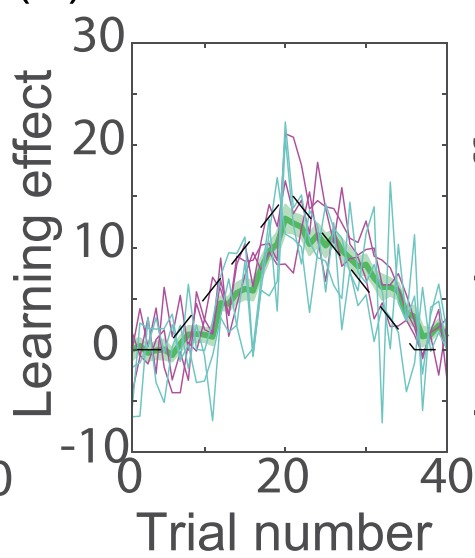

(C)
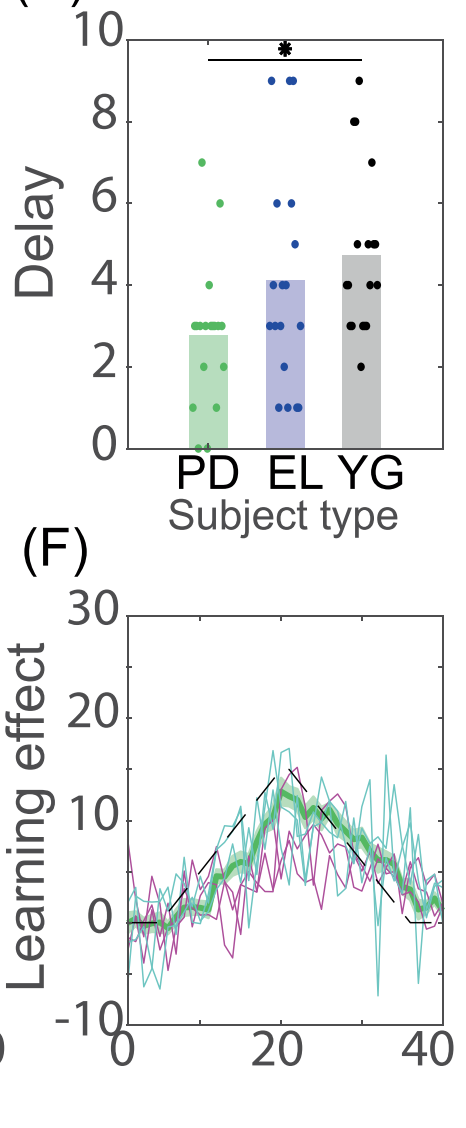

Figure 2. Adaptation effects decomposed into three factors. (A) Amplitude in each subject and group. A larger value indicates a larger adaptation effect. Each dot indicates the amplitude for each subject, and each bar shows the mean amplitude in each group. * and ** indicate significant differences with $\mathrm{p}<0.05$ and $\mathrm{p}<0.01$, respectively (Tukey's post hoc test following one-way ANOVA). (B) Cosine function of the phase for each subject and group. A larger value indicates a smaller phase value, which indicates a similar adaptation curve to the applied perturbation pattern. (C) Delay in each subject and group. A larger value indicates a longer delayed response to the applied perturbation. (D) Typical adaptation curves in the PD subjects whose amplitude was the largest, the second largest, the third largest (magenta solid lines), the third smallest, the second smallest, and the smallest (cyan solid lines). (E) Typical adaptation curves of the PD patients regarding the cosine function of the phase. (F) Typical adaptation curves of the PD patients regarding delays.

participants. In contrast to the assumption, there was no significant group effect $(\mathrm{F}(2,51)=2.58, \mathrm{p}=0.086)$ and no significant difference among the three groups (Fig. 3C,D, $\mathrm{p}=0.43$ between the PD patients $[24.39 \pm 0.60 \mathrm{~mm}$ ] and the elderly individuals $[25.42 \pm 0.57 \mathrm{~mm}], \mathrm{p}=0.0697$ between the PD patients and the young individuals $[26.25 \pm 0.57 \mathrm{~mm}]$, and $\mathrm{p}=0.57$ between the elderly individuals and the young individuals). These results indicate that the feedback response was not a significant factor affecting the adaptation effects.

We further investigated the relationship between the clinical scores and the adaptation effects (Fig. 4). There was no significant correlation between all the recorded attributes and the clinical scores (i.e., age, duration, H\&Y, MMSE, and UPDRS) and the properties of the adaptation effects (i.e., the amplitude and the temporal delay) (Fig. 4, $\mathrm{p}>0.102$ for Pearson's correlation coefficient, and $\mathrm{p}>0.208$ for Spearman's rank correlation coefficient). These results indicate that the recorded attributes and conventional clinical scores were not enough to explain the large amplitude and the small response delays in PD patients.

\section{Discussion}

We investigated the motor adaptation ability inherent in PD patients while decreasing the influence of task switching and the burden of participating in the experiments. To reduce the influence of task switching, we utilized a gradual perturbation that was not noticed by most of the participants. To decrease the burden of participating in the experiments, we used a smart-device-based experiment that enabled us to conduct the experiments anytime and anywhere. The current study revealed that the PD patients possessed a motor adaptation ability that was $20 \%$ larger but not better at minimizing the task errors than the elderly individuals and the young individuals (Figs. 1C-E and 2). The larger adaptation ability was not related to the slow movement speed of the PD patients (Fig. 3A,B), the feedback response to the perturbation (Fig. 3C,D), or the conventional clinical scores (Fig. 4). 

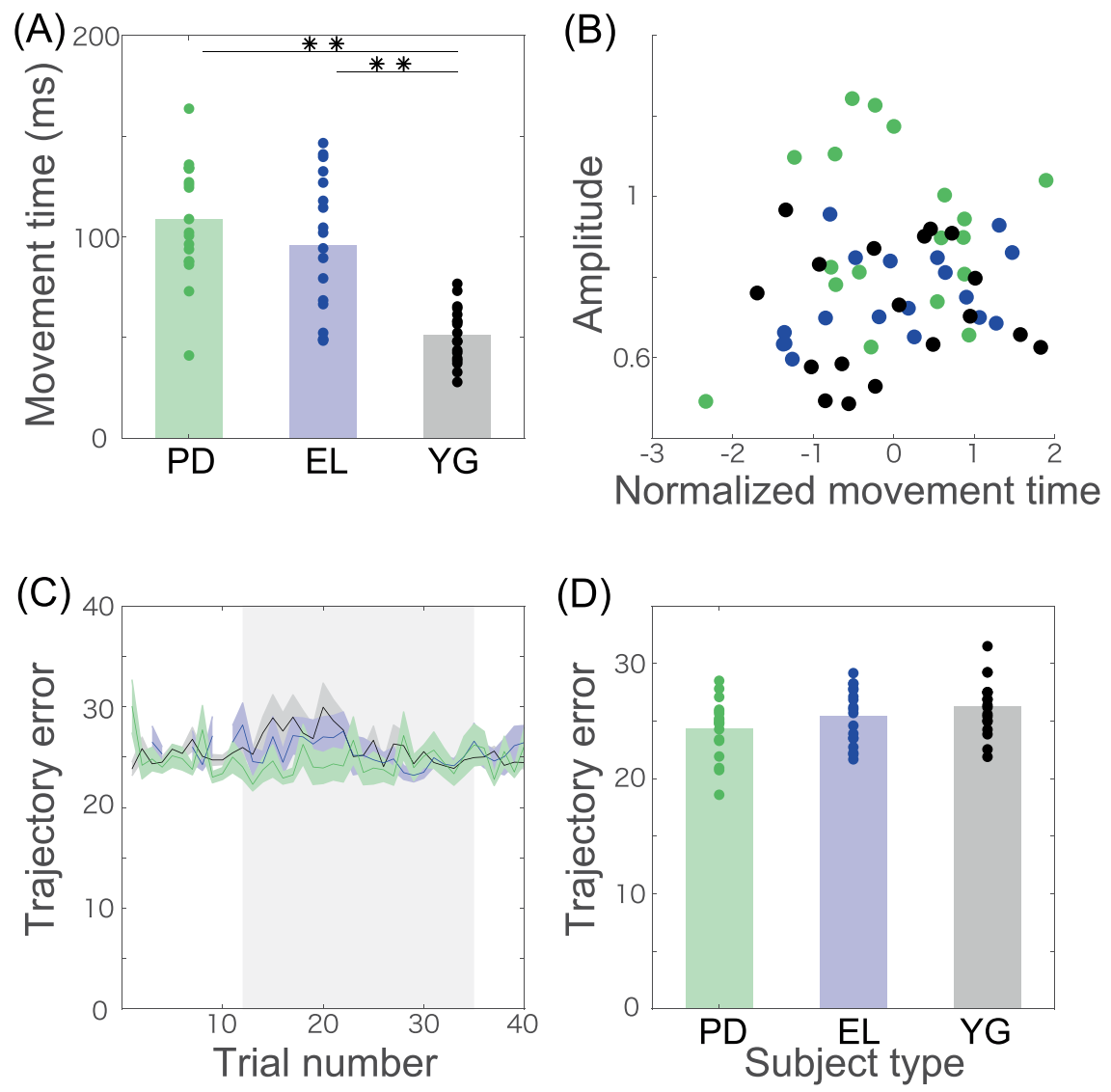

Figure 3. Kinematic factors possibly relating to the adaptation effects. (A) Movement time. ** indicates a significant difference with $\mathrm{p}<0.01$ (Tukey's post hoc test following one-way ANOVA). (B) Relation between the normalized movement time and the amplitude of the adaptation curve. The normalized movement time indicates the modified movement time whose mean and standard deviation are zero and one, respectively, in each group. There was no correlation between the two variables $(\mathrm{r}=0.1517, \mathrm{p}=0.2736)$. (C) Trajectory error. The horizontal axis indicates the trial number, and the vertical axis indicates the trajectory error. The trajectory error was calculated as the squared lateral deviation of the cursor trajectory. The gray shaded area shows the trial numbers where the adaptation effects were significantly different from zero ( $\mathrm{t}$-test $\mathrm{p}<0.01$ [corrected]). (D) Trajectory error averaged across the trials in the gray shaded area in panel (C). There was no difference among the groups ( $\mathrm{p}>0.0697$, Tukey's post hoc test following one-way ANOVA).

The larger adaptation ability originated mostly in the larger amplitude (Fig. 2A) and slightly in the faster response delay (Fig. 2C).

A possible factor for the larger motor adaptation ability in PD patients is compensatory cerebellar function ${ }^{28}$. The cerebellum plays essential roles in motor adaptation ${ }^{1,4}$. Cerebellar ataxia patients have shown deficits in updating the internal model in motor adaptation experiments ${ }^{1,4,5}$. Although it is widely known that PD patients have paretic symptoms related to dopamine or the basal ganglia, a recent finding supported the possibility that they have compensatory cerebellar functions ${ }^{28}$. The compensatory function in PD patients can be supported by not only anatomical connectivities between the cerebellum and basal ganglia ${ }^{29}$ but also resting and functional

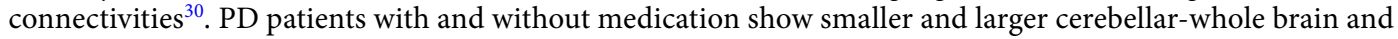
cerebellar-cerebellar resting connectivities compared to healthy controls, respectively ${ }^{30}$. Because we focused on PD patients with medication, their cerebellar-whole brain and cerebellar-cerebellar connectivities were likely smaller than those of the elderly and the young. In other words, some cerebellar contributions in the medicated PD patients could be smaller those of other participants in the current study.

The smaller contribution of the cerebellum resulted in a smaller prediction ability in PD patients because the cerebellum plays a role in predicting perturbation ${ }^{31}$. In particular, our results supported the weaker involvement of the prospective error ${ }^{18}$, the predicted error in the upcoming movements, in the motor adaptation of the PD patients compared to the elderly and the young. Counterintuitively, in the framework of the prospective error, a deficit of prediction ability leads to a large motor adaptation ability. In this framework, the predicted error determines a recruitment pattern of neural units responsible for generating motor commands. The adaptation effects when subjects do not predict the prospective error are larger than when they do predict the error. Without the prediction of the prospective error, the same pattern of neural units is recruited across all the trials, and the adaptation effects are embedded in the pattern in a concentrated manner. With the prediction of the prospective error, a different pattern of neural units is recruited according to the updated prospective error in each trial, and the 

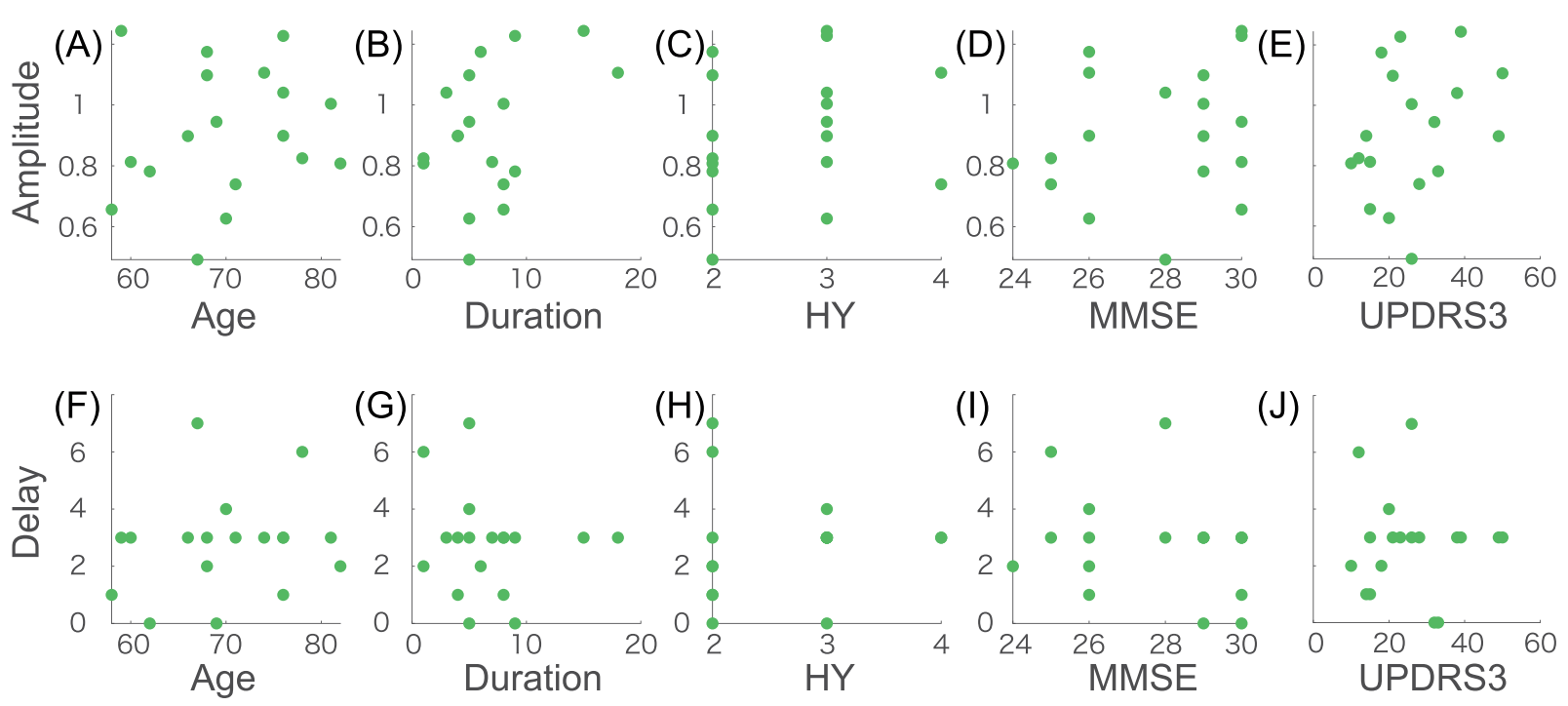

Figure 4. Correlation between the adaptation effects (amplitude and delay) and the clinical scores of the PD patients. (A-E) The relationships between the amplitude and the scores. (F-J) The relationships between the delay and the scores. There was no significant correlation between the adaptation effects and the clinical scores ( $\mathrm{p}>0.1024$ for Pearson's correlation coefficient and $\mathrm{p}>0.2075$ for Spearman's rank correlation coefficient).

adaptation effects are embedded in several patterns in a distributed manner. The adaptation effects embedded in the concentrated population, rather than in the distributed population, show large adaptation effects. A possibility inherent in the current finding is thus that PD patients possess an impaired ability to predict the prospective error. Another study reported a deficit prospective ability in PD patients with medication ${ }^{32}$. Furthermore, this prospective error hypothesis can also explain the lack of savings and anterograde interference (a lower adaptation speed in an interfered task in the A-B paradigm) in PD patients ${ }^{19,20}$. Summarizing, our results likely suggest that the larger, but not better, motor adaptation ability inherent in medicated PD patients would originate from a deficit prospective ability through smaller cerebellar-whole brain and cerebellar-cerebellar connectivities.

Another possible factor for the larger observed motor adaptation ability in PD patients is the reward associated with the motor adaptation task ${ }^{33,34}$. Because dopamine neurons can encode reward information, such as temporal difference error ${ }^{35,36}$, the lack of dopamine neurons in PD patients can affect motor adaptation through a deficit in encoding reward information. In several motor adaptation studies, rewards are associated with accomplishing the performed movements $s^{33,37}$. In our experiments, the controlled cursor hit the target before, during, and after adaptation with a high probability (Fig. 1A). Furthermore, in our previous study, we showed that there was no difference between the motor adaptation with and without the vibration in hitting the target in healthy young adults, which was regarded as with and without the reward associated with the success of the movement ${ }^{11}$. Thus, we can suggest that our results may originate from compensatory cerebellar function rather than a deficit in encoding reward information.

Although the state-space model ${ }^{38-41}$ is a popular method to quantify the adaptation effects in motor adaptation, it is not appropriate in the current study. In this framework, the task error should be minimized. In our current setting, the adaptation effects of PD patients took smaller values than the perturbation sequence until the 24th trial and larger values from the 25 th trial. In this case, the framework of the state-space model predicted that the adaptation effects increased in each trial until the 24th trial; however, in our experimental setting, the learning effects decreased from the 20th trial. Thus, we did not apply the state-space model in our current setting. Our findings suggest the need to improve the state-space model to explain adaptation to gradually applied and vanishing perturbations.

Promising future work using PoMLab would be to investigate motor adaptation ability inherent in several types of patients, such as stroke patients, cerebellar ataxia patients, and Huntington disease patients, or the ability associated with autism spectral disorder, schizophrenia, etc. A cross-syndrome comparison can provide essential knowledge about the neural mechanisms of updating the internal model. Although several studies have investigated the ability of patients $s^{4,5,26,42}$, the experimental setting is different in each study. PoMLab is a cross-platform application and is available for free on our GitHub page (https://github.com/masahiroshinya/PoMLab), which can help researchers, physical therapists, medical doctors, or anyone conduct motor adaptation experiments. Additionally, PoMLab supports conducting motor adaptation experiments anytime and anywhere while decreasing the burden of participating in an experiment.

\section{Data availability}

The datasets analyzed in the current study are available from the corresponding author upon reasonable request.

Received: 24 July 2019; Accepted: 9 February 2020;

Published online: 28 April 2020 


\section{References}

1. Wolpert, D. M., Miall, R. C. \& Kawato, M. Internal models in the cerebellum. Trends in Cog Sci 2, 338-347 (1998).

2. Shadmehr, R. \& Mussa-Ivaldi, F. A. Adaptive representation of dynamics during learning of a motor task. J Neurosci 14, 3208-3224 (1994).

3. Krakauer, J. W., Pine, Z. M., Ghilardi, M. F. \& Ghez, C. Learning of visuomotor transformations for vectorial planning of reaching trajectories. J Neurosci 20, 8916-8924 (2000).

4. Smith, M. A. \& Shadmehr, R. Intact ability to learn internal models of arm dynamics in Huntington's disease but not cerebellar degeneration. J Neurophysiol 93, 2809-2821 (2005).

5. Gibo, T. L., Criscimagna-Hemminger, S. E., Okamura, A. M. \& Bastian, A. J. Cerebellar motor learning: are environment dynamics more important than error size? J Neurophysiol 110, 322-333 (2013).

6. Contreras-Vidal, J. L. \& Buch, E. R. Effects of Parkinson's disease on visuomotor adaptation. Exp Brain Res 150, 25-32 (2003).

7. Messier, J. et al. Visuomotor learning in immersive 3D virtual reality in Parkinson's disease and in aging. Exp Brain Res 179, 457-474 (2007).

8. Taylor, J. A., Krakauer, J. W. \& Ivry, R. B. Explicit and implicit contributions to learning in a sensorimotor adaptation task. J Neurosci 34, 3023-3032 (2014).

9. Butcher, P. A. et al. The cerebellum does more than sensory prediction error-based learning in sensorimotor adaptation tasks. $J$ Neurophysiol 118, 1622-1636 (2017).

10. Kagerer, F. A., Contreras-Vidal, J. L. \& Stelmach, G. E. Adaptation to gradual compared with sudden visuomotor distortions. Exp Brain Res 115, 557-561 (1997).

11. Takiyama, K. \& Shinya, M. Development of Portable Motor Learning Laboratory (PoMLab). PLoS ONE 11, e0157588 (2016).

12. Venkatakrishnan, A., Banquet, J. P., Burnod, Y. \& Contreras-Vidal, J. L. Parkinson's disease differentially affects adaptation to gradual compared to sudden visuomotor distortions. Hum Mov Sci 30, 760-769 (2011).

13. Mongeon, D., Blanchet, P. \& Messier, J. Impact of Parkinson's disease and dopaminergic medication on adaptation to explicit and implicit visuomotor perturbations. Brain and $\operatorname{Cog} \mathbf{8 1}, 271-282(2013)$.

14. Ishii, K., Hayashi, T. \& Takiyama, K. Influence of switching rule on motor learning. Sci Rep 8, 13559 (2018).

15. Furuki, D. \& Takiyama, K. Decomposing motion that changes over time into task-relevant and task-irrelevant components in a data-driven manner: application to motor adaptation in whole-body movements. Sci Rep 9, 7246 (2019).

16. Krakauer, J. W., Ghez, C. \& Ghilardi, M. F. Adaptation to visuomotor transformations: consolidation, interference, and forgetting. $J$ Neurosci 25, 473-478 (2005).

17. Smith, M. A., Ghazizadeh, A. \& Shadmehr, R. Interacting adaptive processes with different timescales underlie short-term motor learning. PLoS Biol 4, e179 (2006).

18. Takiyama, K., Hirashima, M. \& Nozaki, D. Prospective errors determine motor learning. Nat Comm 6, 5925 (2015).

19. Marinelli, L. et al. Learning and consolidation of visuo-motor adaptation in Parkinson's disease. Parkinsonism Relat Disord 15, 6-11 (2009).

20. Leow, L. A., de Rugy, A., Loftus, A. M. \& Hammond, G. Different mechanisms contributing to savings and anterograde interference are impaired in Parkinson's disease. Front Hum Neurosci 7 (2013).

21. Fernandes, H. L., Albert, M. V. \& Kording, K. P. Measuring generalization of visuomotor perturbations in wrist movements using mobile phones. PLoS ONE 6, e20290 (2011).

22. Bedore, C. D., Livermore, J., Lehmann, H. \& Brown, L. E. Comparing three portable, tablet-based visuomotor tasks to laboratory versions: An assessment of test validity. J Concussion 2, 1-15 (2018).

23. Fahn, S., Elton, R. L. Unified rating scale for Parkinson's disease. In: Fahn, S., Marsden, C. D. editors, Recent Developments in Parkinsons Disease, Florham Park. pp. 153-163, 293-304 (1987).

24. Schenkman, M., Zhu, C. W., Cutson, T. M. \& Whetten-Goldstein, K. Longitudinal evaluation of economic and physical impact of Parkinson's disease. Parkinsonism Relat Disord 8, 41-50 (2001).

25. Semrau, J. A., Perlmutter, J. S. \& Thoroughman, K. A. Visuomotor adaptation in Parkinson's disease: effects of perturbation type and medication state. J Neurophysiol 111, 2675-2687 (2014).

26. Miyamoto, H., Kawato, M., Setoyama, T. \& Suzuki, R. Feedback-Error-Learning Neural Network for Trajectory Control of a Robotic Manipulator. Neural Netw 1, 251-265 (1988).

27. Majsak, M. J., Kaminski, T., Gentile, A. M. \& Flanagan, J. R. The reaching movements of patients with Parkinson's disease under self-determined maximal speed and visually cued conditions. Brain 121(4), 755-766 (1998).

28. Mirdamadi, J. L. Cerebellar role in Parkinson's disease. J Neurophysiol 116, 917-919 (2016).

29. Hoshi, E., Tremblay, L., Feger, J., Carras, P. L. \& Strick, P. L. The cerebellum communicates with the basal ganglia. Nat Neurosci 8, 1491-1493 (2005).

30. Festini, S. B. et al. Front Hum Neurosci 9, 214 (2015).

31. Scheidt, R. A. et al. Remembering forward: Neural correlates of memory and prediction in human motor adaptation. NeuroImage 59, 582-600 (2012).

32. Saito, N. et al. Altered awareness of action in Parkinson's disease: evaluations by explicit and implicit measures. Sci Rep 7, 8019 (2017).

33. Izawa, J. \& Shadmehr, R. Learning from Sensory and Reward Prediction Errors during Motor Adaptation. PLoS Comput Biol 7, 11 (2011).

34. Galea, J. M., Mallia, E., Rothwell, J. \& Diedrichsen, J. The dissociable effects of punishment and reward on motor learning. Nat Neurosci 18, 597-602 (2015).

35. Montague, P. R., Dayan, P. \& Sejnowski, T. J. A framework for mesencephalic dopamine systems based on predictive Hebbian learning. J Neurosci 16, 1936-1947 (1996).

36. Schultz, W., Dayan, P. \& Montague, P. R. A neural substrate of prediction and reward. Science 275, 1593-1599 (1997).

37. Pekny, S. E., Izawa, J. \& Shadmehr, R. Reward-Dependent Modulation of Movement Variability. J Neurosci 35, 4015-4024 (2015).

38. Thoroughman, K. A. \& Shadmehr, R. Learning of action through adaptive combination of motor primitives. Nature 407, 742-747 (2000).

39. Scheidt, R. A., Dingwell, J. B. \& Mussa-Ivaldi, F. A. Learning to move amid uncertainty. J Neurophysiol 86, 971-985 (2001).

40. Takiyama, K. \& Sakai, Y. Balanced motor primitive can explain generalization of motor learning effects between unimanual and bimanual movements. Sci Rep, 1-10 (2016).

41. Takiyama, K. Context-dependent memory decay is evidence of effort minimization in motor learning: a computational study. Front Comput Neurosci 9(4), 1-10 (2015).

42. Patton, J. L., Stoykov, M. E., Kovic, M. \& Mussa-Ivaldi, F. A. Evaluation of robotic training forces that either enhance or reduce error in chronic hemiparetic stroke survivors. Exp Brain Res 168, 368-383 (2006).

\section{Acknowledgements}

We thank the staff at Kakeyu Rehabilitation Hospital for their kind support for this research. In particular, we thank Youichi Maruyama and the physical therapists at the hospital. We also thank Shin-ichi Muramatsu for his help and Shintaro Uehara for his helpful comments. We acknowledge support from the Nakajima Foundation, the Kayamori Foundation of Informational Science, a Novartis Pharma Research Grant, a Grant-in-Aid for Young Scientists (18K17894), and a Grant-in-Aid for Challenging Exploratory Research (16K12988). 


\section{Author contributions}

K.T. and M.S. designed the experiments; T.S., T.S., and H.O. performed the experiments; K.T. performed the analyses and wrote the manuscript;. T.K. oversaw the manuscript.

\section{Competing interests}

The authors declare no competing interests.

\section{Additional information}

Correspondence and requests for materials should be addressed to K.T.

Reprints and permissions information is available at www.nature.com/reprints.

Publisher's note Springer Nature remains neutral with regard to jurisdictional claims in published maps and institutional affiliations.

(c) (i) Open Access This article is licensed under a Creative Commons Attribution 4.0 International License, which permits use, sharing, adaptation, distribution and reproduction in any medium or format, as long as you give appropriate credit to the original author(s) and the source, provide a link to the Creative Commons license, and indicate if changes were made. The images or other third party material in this article are included in the article's Creative Commons license, unless indicated otherwise in a credit line to the material. If material is not included in the article's Creative Commons license and your intended use is not permitted by statutory regulation or exceeds the permitted use, you will need to obtain permission directly from the copyright holder. To view a copy of this license, visit http://creativecommons.org/licenses/by/4.0/.

(C) The Author(s) 2020 